\title{
FINITE TIME RUIN PROBLEMS FOR PERTURBED EXPERIENCE RATING AND CONNECTION WITH DISCOUNTING RISK MODELS
}

\author{
By F. Abikhalil \\ Université Libre de Bruxelles
}

\begin{abstract}
We consider a generalisation of a risk process under experience rating when the aggregation of claims up to time $t$ is a Brownian motion (B.M.) with a drift. We prove that the distribution of ruin before time $t$ is equivalent to the distribution of the first passage time of B.M. for parabolic boundary.

Using Wald identity for continuous time we give an explicit formula for this distribution. A connection is made with discounting risk model when the income process is a diffusion.

When the aggregation of claims is a mixture of B.M. and compound Poisson process, we give (using Gerber's result 1973) an upper bound for the distribution of finite time ruin probability.
\end{abstract}

\section{KEYWORDS}

Ruin probability, Brownian motion, Martingale Ornstein-Ohlebeck process.

\section{INTRODUCTION}

The purpose of this paper is to analyse the ruin probabilities (finite- and infinitetime horizon) for a risk process, where premiums are collected continuously on the basis of the "perturbed" experience rating model.

The principle of the experience rating is to adjust premiums by taking into account previous information. Nevertheless, there is a difference between examining premiums in a theoretical way and how they are applied in reality.

Actually, in practice, the insurer uses "some kind" of experience rating principle and takes into account, let us say, indirect influence factors like, for example:

(1) Uncertainty of inflation;

(2) Time lags between the calculation of premiums and the collection of data concerning claims.

(3) Uncertainty due to a lack of precise knowledge about economic activity.

RANTALA (1982), in his studies of the insurance industry in Finland, suggested (see section 2.2. "models for premiums fluctuation") to add to the experience rating model (for the discrete case) a "noise" term, which seems to be a good approximation to real situations.

Following this idea, in order to take into account the indirect influence factors discussed above, we will add a perturbation by introducing a Brownian motion (B.M.) for the continuous case considered here, and will say that we have a perturbed experience rating.

ASTIN BULLETIN Vol 16, No 1 
Our analyses (Section 4) will rely on a fundamental theorem of SHEPP (1967). For convenience and to make the paper self-contained, this theorem is restated in the appendix.

This paper is organised as follows. In Section 2 and 3 we describe the risk process under "perturbed experience rating".

Section 4 is devoted to analyse the ruin probabilities in finite and infinite-times intervals, when the aggregation of claims up to time $t,\{S(t), t \geqslant 0\}$ is a B.M. with a drift. A connection with classical risk process with discounting is done.

Finally, in Section 5, we apply Gerber's result (1973) to give an upper bound for the ruin probability before $t$, when $\{S(t), t \geqslant 0\}$ is a linear combination of compound poisson process and a B.M. with a drift.

\section{DESCRIPTION OF THE RISK PROCESS AND NOTATIONS}

We consider a risk process in which the total premium received in the time-interval $[0, t]$ is denoted by $P(t)$, and we represent by $\{S(t), t \geqslant 0\}$ the aggregation of claims up to time $t$. We assume that the process $\{P(t), t \geqslant 0\}$ and $\{S(t), t \geqslant 0\}$ are Markovian and defined on a probability space $(\Omega, F, P)$.

Finally, let $\{Z(t), t \geqslant 0\}$ be the surplus process of a company, and write $x$ for $Z(0)$. We have:

$$
Z(t)=x+P(t)-S(t), \quad t \geqslant 0
$$

\section{PERTURBED EXPERIENCE RATING AND THE CORRESPONDING SURPLUS PROCESS}

Consider a risk process satisfying (1) where each element of premium is modified by refund or surcharge according to the stochastic differential equation:

$$
d P(t)=(p-k(P(t)-S(t)) d t+\sigma d W(t), \quad t \geqslant 0
$$

with $P(0)=0$ a.s. and where:

(i) $p$ is the base premium constant rate

(ii) $\{W(t), t \geqslant 0\}$ is a standard B.M. process independent of $\{S(t), t \geqslant 0\}$

(iii) a positive constant, $k$ being the "experience rating factor" $(0<k<1$, in general).

Equation (2) is linear stochastic differential equation. Using classical result from GihmaN and SKorohod (1972), its solution is

$$
P(t)=e^{-k t}\left[\frac{p}{k}\left(e^{k t}-1\right)+k \int_{0}^{t} e^{k s} S(s) d s+\sigma X(t)\right], \quad t \geqslant 0
$$

where

$$
X(t)=\int_{0}^{t} e^{k s} d W(s), \quad t \geqslant 0
$$


Substituting (3) in (1), $\mathcal{Z}(t)$ becomes

(5) $Z(t)=x+\frac{P}{k}\left(1-e^{-k t}\right)+k e^{-k t} \int_{0}^{t} e^{k s} S(s) d s+\sigma e^{-k t} X(t)-S(t), \quad t \geqslant 0$

In order to characterise and reduce this expression we have the following two propositions.

Proposition 1. $\{X(t), t \geqslant 0\}$ is a gaussian process with zero mean and with covariance:

$$
\operatorname{cov}(X(s), X(t))=\int_{0}^{\min (s, t)} e^{2 k u} d u=\frac{1}{2 k}\left(e^{2 k(\min (t, s)}-1\right) .
$$

PRoof. The fact that $X(t)$ is gaussian with zero means is obvious. For relation (6), it suffices to use the well-known property:

$$
E\left[\int_{a}^{b} f(t) d W(t) \cdot \int_{a}^{c} g(t) d W(t)\right]=\int_{a}^{\min (b, c)} f(t) \cdot g(t) d t
$$

where in this formula $f$ and $g$ are assumed to be continuously differentiable on the indicated intervals of integration. The proposition follows then by an elementary computation.

Assume now that $\{S(t), t \geqslant 0\}$ is stationary, independent increments, finite variance with $S(0)=0$ a.s. and belonging to $D[0, \infty)$ where $D[0, \infty)$ denotes the space of functions on $[0, \infty)$ that are right-continuous and have left-hand limits, we have

Proposition 2. The process $\left\{\int_{0}^{t} e^{k s} d S(s), t \geqslant 0\right\}$ is well defined, a.s. finite, and every sample path satisfies the following relation:

$$
\int_{0}^{t} e^{k s} d S(s)=e^{k t} S(t)-k \int_{0}^{t} e^{k s} S(s) d s
$$

Proof. See Harrison (1977) Proposition 2.1.

Corollary. Under the assumption of proposition 2 and substituting (8) in (5) the surplus process can be expressed as:

$$
Z(t)=x+\frac{p}{k}\left(1-e^{-k t}\right)-e^{-k t} \int_{0}^{t} e^{k s} d S(s)+e^{-k t} \sigma X(t), \quad t \geqslant 0
$$

4. RUIN PROBABILITIES WHEN $\{S(t), t \geqslant 0\}$ IS A B.M. WITH A DRIFT

\subsection{Description and Characterisation of the Surplus Process}

Assume moreover that $S(t)$ satisfies the differential (stochastic) equation;

$$
d S(t)=m d t+\sigma_{1} d W_{1}(t)
$$


where $m$ is a positive constant and $\left\{W_{1}(t), t \geqslant 0\right\}$ is a standard B.M. independent of $\{W(t), t \geqslant 0\}$.

Then the relation (9) gives:

$$
Z(t)=x+\frac{p}{k}\left(1-e^{-k t}\right)-e^{-k t} \int_{0}^{t} e^{k s} m d s+e^{-k t}\left(\sigma_{1} X_{1}(t)+\sigma X(t)\right)
$$

where we define, as before

$$
X_{1}(t)=-\int_{0}^{t} e^{k s} d W_{1}(s)
$$

From proposition $1,\left\{X_{1}(t), t \geqslant 0\right\}$, is a gaussian process independent of $\{X(t), t \geqslant$ 0 \} with zero mean and as covariance function;

$$
\operatorname{cov}\left(X_{1}(s), X_{1}(t)\right)=\frac{1}{2 k}\left(e^{2 k(\min (t, s))}-1\right) .
$$

It is well-known that the sum of two independent gaussian processes is a gaussian one. So we can write:

$$
\sigma_{1} X_{1}(t)+\sigma X(t)=\hat{\sigma} \hat{X}(t)
$$

where

(i) $\{\hat{X}(t), t \geqslant 0\}$ is a gaussian process with zero mean and having the same covariance function as $\left\{X_{1}(t), t \geqslant 0\right\}$

$$
\hat{\sigma}^{2}=\sigma_{1}^{2}+\sigma^{2}
$$

Consequently;

$$
Z(t)=x+\frac{\mu}{k}\left(1-e^{-k t}\right)+\hat{\sigma} e^{-k t} \hat{X}(t), \quad t \geqslant 0
$$

where $\mu=p-m>0$

Now let $\mu^{*}=\mu+k x$.

Proposition 3. If $\{S(t), t \geqslant 0\}$ is a B.M. with a drift $m$, the process $\{Z(t), t \geqslant 0\}$, is an Ornstein-Uhlenbeck process with a drift $\mu(y)=\mu^{*}-k y$ and an infinitesimal variance $\sigma^{2}(y)=\hat{\sigma}^{2}$.

Proof. It is clear that $Z(t)$ is a gaussian and has continuous sample paths with independent increments.

Write

$$
Z(t)=e^{-k t} \zeta(t), \quad t \geqslant 0
$$

with

$$
\zeta(t)=x e^{k t}+\frac{\mu}{k}\left(e^{k t}-1\right)+\hat{\sigma} \hat{X}(t), \quad t \geqslant 0 .
$$

By differentiating (15) we get

$$
d Z(t)=-k Z(t) d t+\mu^{*} d t+\hat{\sigma} d W_{2}(t), \quad t \geqslant 0
$$


where $\left\{W_{2}(t), t \geqslant 0\right\}$ is a standard B.M. From this, the proposition follows immediately.

REMARK. We will call $\zeta(t)$, the modified surplus process, and will represent it by the compounding B.M.:

$$
\zeta(t)=x e^{k t}+\frac{\mu}{k}\left(e^{k t}-1\right)+\frac{\hat{\sigma}}{\sqrt{2 k}} W\left(e^{2 k t}-1\right), \quad t \geqslant 0 .
$$

\subsection{The Ruin Functions}

We are interested in the random variable "time of ruin" defined as usual by:

$$
T=\inf \{t \geqslant 0: Z(t) \leqslant 0\} .
$$

Introduce the ruin functions, respectively on finite and infinite-time horizons:

$$
\begin{aligned}
\psi(x, t) & =P[T \leqslant t / Z(0)=x] \\
\psi(x) & =P[T<\infty / Z(0)=x] .
\end{aligned}
$$

\subsubsection{The Ultimate Ruin is Certain}

It is obvious that $Z(t)$ and $\zeta(t)$ hit zero at the same random time $T$, so at this time and from (18),

$$
x e^{k T}+\frac{\mu}{k}\left(e^{k T}-1\right)+\frac{\hat{\sigma}}{\sqrt{2 k}} W\left(e^{2 k T}-1\right)=0 .
$$

Let $v$ be the scale change in time defined by $v=e^{2 k t}-1$ and let $v^{*}=e^{2 k T}-1$.

In terms of $v(22)$ gives: $W\left(v^{*}\right)=\xi\left(v^{*}\right)$ where

$$
\xi(v)=\alpha-\gamma \sqrt{v+1}, \quad v \geqslant 0 .
$$

$\alpha, \gamma$ beine two positive constants defined by

Obviously

$$
\alpha=\sqrt{\frac{2}{k}} \frac{\mu}{\hat{\sigma}} \quad \text { and } \quad \gamma=\sqrt{\frac{2}{k}} \frac{1}{\hat{\sigma}}(k x+\mu) .
$$

(i) $\xi(v)$ is continuous

(ii) $\xi(0)=\alpha-\gamma=-x \sqrt{2 k} / \hat{\sigma}<0$

(iii) $\xi(v) / v \rightarrow_{v \rightarrow \infty} 0$

We can then apply Shepp's result (see appendix, corollary A) for the function (23) and deduce that the ultimate ruin is certain.

\subsubsection{Ruin Probability on Finite-Time Interval}

Our objective in this section is to study the function $\psi(x, t)$, which we assume to have density $p(t)$ (the argument $x$ is omitted), or in terms of $v$, we are interested in the density $g(v)$, where $g(v) d v=p(t) d t$. 
From theorem A (appendix) we have

$$
\int_{0}^{\infty} e^{-\lambda \alpha+\lambda \gamma \sqrt{v+1}-\lambda^{2}(v / 2)} g(v) d v=1, \quad \lambda>0 .
$$

then following the method used by SHEPP (1967) or DANIELS (1969), for $\operatorname{Re}(s)>0$,

$$
\text { (25) } \begin{aligned}
\int_{0}^{\infty} \lambda^{s-1} e^{\lambda \alpha-\lambda^{2} / 2} d \lambda & =\int_{0}^{\infty} \lambda^{s-1} e^{\lambda \alpha-\lambda^{2} / 2} d \lambda \int_{0}^{\infty} e^{-\lambda \alpha+\lambda \gamma \sqrt{v+1}-\lambda^{2}(v / 2)} g(v) d v \\
& =\int_{0}^{\infty} y^{s-1} e^{\gamma y-\frac{1}{2} y^{2}} d y \int_{0}^{\infty}(v+1)^{-s / 2} g(v) d v
\end{aligned}
$$

where $y=\lambda \sqrt{v+1}$

Relation (25) gives the Mellin transform of $g(v)$, or the Laplace transform of the first passage time density of the process $Z(t)\left(\mathrm{cf} . v+1=e^{2 k t}\right)$, which means that if we set $\tau=k t$, this density is $h(\tau)$ where $h(\tau) d \tau=g(v) d v$ and consequently (25) becomes:

$$
h^{*}(s)=\int_{0}^{\infty} e^{-s \tau} h(\tau) d \tau=\frac{I(s, \alpha)}{I(s, \gamma)}
$$

where

$$
I(s, \alpha)=\frac{1}{\Gamma(s)} \int_{0}^{\infty} y^{s-1} e^{\alpha y-\frac{1}{2} y^{2}} d y=e^{-\frac{1}{4} \alpha^{2}} D_{-s}(-\alpha)
$$

in terms of the parabolic cylinder function.

KEILSON and Ross (1975) prepared tables of $h(t)$ which give numerically the simple zero, $-\beta_{j}$, of the denominator of $(26)$ on the negative real axis with the appropriate coefficients

$$
\sigma_{j}(\alpha, \beta)=\left\{I(s, \alpha) / \frac{\partial}{\partial s} I(s, \alpha)\right\} s=-\beta,
$$

of the simple spectral decomposition

$$
h(\tau)=\sum_{J=1}^{\infty} \sigma_{j}(\alpha, \beta) e^{-\beta, \tau} .
$$

The preceding result can be summarised in the following proposition.

Proposition 4.

$$
\begin{aligned}
\psi(x, t) & =\int_{0}^{t k} h(\tau) d \tau \\
& =\sum_{J=1}^{\infty} \frac{\sigma_{J}(\alpha, \beta)}{\beta_{J}}\left(1-e^{-\beta, t k}\right) .
\end{aligned}
$$

Where $\sigma_{J}(\alpha, \beta)$ and $\beta_{J}$ are defined above. 


\subsection{Connection with Classical Discounted Risk Process}

In his paper, HARRISON (1977) studied the ultimate ruin probability for a classical model with interest rate $\beta$. The basic equation for the surplus process denoted here by $\tilde{Z}(t)$, was (see example 3.3. of HARRISON 1977)

$$
\tilde{Z}(t)=e^{\beta t}\left[x+\frac{\tilde{\mu}}{\beta}\left(1-e^{-\beta t}\right)+\tilde{\sigma} \int_{0}^{t} e^{-\beta s} d W_{3}(s)\right], \quad t \geqslant 0
$$

where $\tilde{\mu}, \tilde{\sigma}$ are two positive constants and $\left\{W_{3}(t), t \geqslant 0\right\}$ a standard B.M.

This process is a diffusion, and its infinitesimal mean and variance are $\tilde{\mu}(y)=$ $\tilde{\mu}+y$ and $\tilde{\sigma}^{2}(y)=\tilde{\sigma}^{2}, y \in \mathbb{R}$, respectively.

The modified process, (the square-bracketed term in (31) denoted by

$$
\tilde{\zeta}(t)=x+\frac{\tilde{\mu}}{\beta}\left(1-e^{-\beta t}\right)+\sqrt{\frac{\tilde{\sigma}}{2 \beta}} W_{4}\left(1-e^{-2 \beta t}\right), \quad t \geqslant 0
$$

where $\left\{W_{4}(t), t \geqslant 0\right\}$ is a standard B.M.

In this case, Harrison showed that the ultimate ruin is not certain and its probability is:

$$
\tilde{\psi}(x)=\frac{1-\Phi(a x+b)}{1-\Phi(b)}
$$

where $a$ and $b$ are two positive constants defined by:

$$
a=\sqrt{\frac{2 \beta}{\tilde{\sigma}^{2}}} \text { and } b=\frac{a \tilde{\mu}}{\beta}
$$

and $\Phi(\cdot)$ being the standardised normal distribution.

This result is not surprising, because if we make in (32) the scale change in time $\tilde{v}=\left(1-e^{-2 \beta t}\right)$, the process $\{\tilde{\zeta}(t), t \geqslant 0\}$ hits zero when

$$
W_{4}(\tilde{v})=\left(x+\frac{\tilde{\mu}}{\beta}\right)+\frac{\tilde{\mu}}{\beta} \sqrt{1-\tilde{v}}, \quad 0 \leqslant \tilde{v} \leqslant 1
$$

so in terms of $\tilde{v}$, the B.M. stops at $\tilde{v}=1$ and it is not certain that $W_{4}(\tilde{v})$ will hit the parabolic boundary before $\tilde{v}=1$.

\section{IMPORTANT REMARKS}

(1) The rescaling of B.M. representation is a very delicate operation. We cannot make it for a process like $\tilde{Z}(t)$ as was done in HARRISON (1977). The reason is that the covariance of the integral representation of $\tilde{Z}(t)$, see equation (31) is not the same as that of the rescaling B.M. (see equation (18) in HARrison 1977).

(2) The difference between the two processes $(\{Z(t), t \geqslant 0\}$ is characterised as being a B.M. plus an elastic force that pulls the process back toward zero with a constant proportionality to the current absolute positions $k(k>0)$. While, $\{\tilde{Z}(t), t \geqslant 0\}$ is characterised similarly but the elastic force is a repulsive one pushing the process away from zero (constant proportionality $\beta)$. 
5. UPPER BOUND FOR RUIN PROBABILITY WHEN THE AGGREGATION OF CLAIMS IS A COMPOUND POISSON PROCESS

Let $S(t)$ be a compound Poisson process; we can write,

$$
S(t)=\sum_{i=1}^{N(t)} A_{i}, \quad t \geqslant 0
$$

where $\left\{\boldsymbol{A}_{t}\right\}_{1 \geqslant 1}$ is a sequence of positive independent, identically distributed random variables with a common distribution function $F(\cdot)$, and $\{N(t), t \geqslant 0\}$ is a Poisson stochastic process, independent of the $\left\{A_{t}\right\}_{1 \geqslant 1}$, having parameter $\lambda$.

Moreover, we assume $S(t)$ independent of $\{X(t), t \geqslant 0\}$, defined in section 3 . In the context of classical risk theory:

$A_{1}$ denotes the amount of the $i$ th claim $(i=1,2, \ldots)$ and

$N(t)$ represents the total number of claims occurring in the time-interval $[0, t]$.

Thus, the Riemann-Stieltjes integral $\int_{0}^{t} e^{k s} d S(s)$ becomes:

$$
\sum_{i=1}^{N(t)} e^{k t_{i}} A_{i}
$$

where $t_{1}, t_{2}, \ldots$, denote the times at which claims occur.

The surplus process (9) is now:

$$
Z(t)=x+\frac{p}{k}\left(1-e^{-k t}\right)-e^{-k t} \sum_{i=1}^{N(t)} e^{k t} A_{t}+\sigma e^{-k t} X(t), \quad t \geqslant 0
$$

or equivalently

$$
\begin{aligned}
Z(t) & =e^{-k t}\left[x e^{k t}+\frac{p}{k}\left(e^{k t}-1\right)+\sigma X(t)-\sum_{t=1}^{N(t)} e^{k t} \cdot A_{t}\right] \\
& =e^{-k t}\left[\tilde{X}(t)-X^{*}(t)\right], \quad t \geqslant 0
\end{aligned}
$$

where

$$
\begin{array}{rlrl}
\tilde{X}(t) & =x e^{k t}+\frac{p}{k}\left(e^{k t}-1\right)+\sigma X(t), & & t \geqslant 0 \\
X^{*}(t) & =\sum_{t=1}^{N(t)} e^{k t} A_{t}, & t \geqslant 0 .
\end{array}
$$

As before, $\{X(t), t \geqslant 0\}$ is a gaussian process with independent increments with

$$
\begin{aligned}
& \mathbb{E}[\tilde{X}(t)]=x e^{k t}+\frac{p}{k}\left(e^{k t}-1\right), \\
& \operatorname{var}[\tilde{X}(t)]=\frac{\sigma^{2}}{2 k}\left(e^{2 k t}-1\right)
\end{aligned}
$$


and with as moment generating function:

$$
M(r, t)=\exp \left\{-r\left[x e^{k t}+\frac{p}{k}\left(e^{k t}-1\right)\right]+\frac{r^{2}}{2} \frac{\sigma^{2}}{2 k}\left(e^{2 k t}-1\right)\right\}
$$

Consider $Z^{*}(t)=e^{k t} Z(t)-x$.

Obviously $Z^{*}(t)$ is a process with independent increments, then we can apply Gerber's result (1973) to calculate an upper bound for $\Psi(x ; t)$.

In our case, we have

$$
\Psi(x, t) \leqslant \min _{r} e^{-r x} \max _{0 \leqslant s \leqslant t} \mathbb{E}\left[e^{-r Z^{*}(s)}\right] .
$$

Since $\{X(t), t \geqslant 0\}$ and $\left\{X^{*}(t), t \geqslant 0\right\}$ are independent, using (43), (44) becomes: (45) $\Psi(x, t) \leqslant \min _{r} e^{-r x} \max _{0 \leqslant s \leqslant t} \exp \left\{-r\left(x+\frac{p}{k}\right)\left(e^{k s}-1\right)+\frac{\sigma^{2}}{4 k}\left(e^{2 k s}-1\right) r^{2}+K^{*}(r, s)\right\}$ where $K^{*}(r, s)$ is the cumulant generating function of $X^{*}(s)$.

From C. G. TAYLOR's paper (1979) we have

$$
K^{*}(r, s)=\frac{\lambda}{k} \int_{r}^{r e^{k i}} \frac{\delta(u)-1}{u} d u
$$

where $\delta(u)$ denotes the moment generating function associated with $F(\cdot)$. As in TAYLOR's paper (1979) we can only consider values of $r$ such that $r>r^{*}(t)$ with $r^{*}(t)$ being the unique real and positive solution of

$$
-r\left(x+\frac{p}{k}\right)\left(e^{k t}-1\right)+\frac{\sigma^{2}}{4 k}\left(e^{2 k t}-1\right) r^{2}+K^{*}(r, t)=0 .
$$

Then

(48) $\Psi(x, t) \leqslant \min _{r \geqslant r^{*}(t)} \exp \left[-r x-r\left(x+\frac{p}{k}\right)\left(e^{k t}-1\right)+\frac{\sigma^{2}}{4 k}\left(e^{2 k t}-1\right) r^{2}+K^{*}(r, t)\right]$

REMARK. If we take for $S(t)$ a linear combination of a compound Poisson process and a B.M. with a drift (but independent), the whole analysis, in this section is still valid.

The numerical treatment of proposition 4 and equation (48), with comparison with other models (JANSSEN and DELFOSSE 1982) will be developed in a forthcoming paper.

\section{ACKNOWLEDGMENTS}

I am very grateful to Professors J. Janssen and G. Louchard for many helpful discussions and comments, to $\mathrm{M}$. Gassner for her help with translations.

\section{APPENDIX I}

FUNDAMENTAL WALD IDENTITY IN CONTINUOUS-TIME

Let $f(t), t \geqslant 0$ be a continuous function and let $\tau$ be the first time $t$, such that $W(t)=f(t)$ where $\{W(t), t \geqslant 0\}$ is a standard B.M., and let $F(t)=\mathbb{P}(\tau \leqslant t)$. 
Theorem A. If: (1) $\lambda>0$, (2) $f(0)>0$, (3) $f(t) / t \rightarrow 0$ as $t \rightarrow \infty$, then

$$
\int_{0}^{\infty} e^{\lambda f(t)-\lambda^{2}(t / 2)} d F(t)=1 .
$$

Proof (see SHEPP, 1969). Actually, this result is a direct consequence of the fact that the process $\left\{e^{\lambda W(t)-\lambda^{2}(t / 2)}, t \geqslant 0\right\}$ is a martingale.

COROllary A. Let $f(t)=c \sqrt{b+t}-u$ and suppose $u<c \sqrt{b}$

$$
\mathbb{P}[\tau \geqslant t]=\int_{0}^{\infty} d F(t)=1 .
$$

Proof. From (A.1) as $\lambda \rightarrow 0$ the integrand goes to one dominatedly.

\section{APPENDIX II}

In this appendix we give numerical values to illustrate the upper bound in (48); when the distribution of individual claim size is negative exponential, i.e. that

$$
K^{*}(r, t)=\frac{1}{k} \log \left\{\frac{1-r}{1-r e^{k t}}\right\} .
$$

In (49), we assumed that the expected number of claims for unit time is equal to unity.

For $x=\sigma=1, p=1.3$ and various values of $t$ and $k$, we find:

\begin{tabular}{lllll}
$t / k$ & \multicolumn{1}{c}{0.05} & \multicolumn{1}{c}{0.10} & \multicolumn{1}{c}{0.15} & \multicolumn{1}{c}{0.20} \\
\hline 0.1 & $1.47001739 \mathrm{E}-03$ & $1.428926 \mathrm{E}-03$ & $1.38886793 \mathrm{E}-03$ & $1.34982067 \mathrm{E}-03$ \\
0.2 & 0.144325801 & 0.0139840728 & 0.0135472572 & 0.0131219139 \\
0.25 & 0.021223472 & 0.0205326385 & 0.0198601574 & 0.019205716 \\
0.3 & 0.0263070499 & 0.0254139702 & 0.0245450083 & 0.0236998074 \\
0.35 & 0.0295210032 & 0.0284802841 & 0.0274679932 & 0.026483791 \\
0.5 & 0.0305188815 & 0.0293484819 & 0.028208696 & 0.0270998241 \\
0.9 & 0.792848419 & 0.787248899 & 0.781640129 & 0.776030215 \\
0.1 & 0.799357953 & 0.793401234 & 0.787431127 & 0.781457787 \\
1.5 & 0.818592853 & 0.811100702 & 0.803582347 & 0.796061988 \\
2 & 0.826321282 & 0.81754902 & 0.808761104 & 0.80000189
\end{tabular}

\section{REFERENCES}

ARNOLD, L. (1974) Stochastic Differential Equations. Wiley: New York.

Daniels, D. A. (1979) The Minimum of a Stationary Markov Process Superimposed on a U-Shaped Tend. J. Appl. Prob. 6, 399-408.

DUNFORD, N. and SCHWARTZ, J. (1958) Linear Operators. Interscience Publishers: New-York.

Gerber, H. (1973) Martingales in Risk Theory. Mitteilungen der Vereinigung Schweizerischer Versicherungsmathematiker 73, 205-216.

Gerber, H. (1975) The Surplus Process as a Fair Game Utilitywise. The Astin Bulletin 8, 307-322. GIHMAN and SKOROHOD (1972) Stochastic Differential Equations. Springer Verlag.

Harrison, J. M. (1977) Ruin Problems with Compounding Assets. Stochastic Processes and their Applications 5 (1). 
JANSSEN, J. and DELFOSSE, PH. (1982) Some Numerical Aspects in Transient Risk Theory. The Astin Bulletin 13, 99-103.

Pentikainen, T. (1982) Solvency of Insurers and Equalization Reserves, Vol. I. General Aspects. Insurance Publishing Company Ltd.: Helsinki.

Rentala, J. (1982) Solvency of Insurers and Equalization Reserves, Vol. II. Risk Theoretical Model. Insurance Publishing Company Ltd.: Helsinki.

Ruohonen, M. (1980) On the Probability of Ruin of Risk Process Approximated by a Diffusion Process. Scand. Actuarial J. 113-120.

SHEPP, L. A. (1969) Explicit Solutions to Some Problems of Optimal Stopping. Annals of Mathematical Statistics $\mathbf{4 0}$.

TAYLOR, G. C. (1979) Probability of Ruin under inflationary Conditions or under Experience Rating. The Astin Bulletin 10, 149-162.

\section{F. Abikhalil}

Université Libre de Bruxelles, École de Commerce en CEME, CP135, Av. F. D. Roosevelt, 1050 Bruxelles, Belgium. 